\title{
La redéfinition des rôles du traducteur de théâtre face à l'essor du surtitrage
}

\section{The redefinition of the roles of the theater translator in the light of surtitling practices}

\author{
Małgorzata Czubińska \\ Université Adam Mickiewicz de Poznań \\ malgorp@amu.edu.pl
}

\begin{abstract}
During the first two decades of the twenty-first century, we can observe an unprecedented shift in the practice of theatrical translation and a revision of the role of the theater translator. Nowadays, scenic translation methods, including surtitling, put in question the traditional perception of the key concepts of translatology such as translator, equivalence, translation process and methods or even the text. In this paper, based on the theoretical works, the voices of the researchers and theater professionals, the author discusses the status and roles of the theatrical translator as well as the challenges of his activity in the context of the modern theories of theater translation.
\end{abstract}

Keywords: theatrical translation, scenic translation, surtitling, audiovisual translation

\section{INTRODUCTION}

La société moderne est exposée à un nombre inédit de changements provoqués par le développement technologique et ce qui en découle - la révolution au niveau des moyens de communication sociale. Cette évolution constante, ayant une incidence sur les modes de vie des individus, elle trouve son reflet dans le domaine de création artistique, notamment le théâtre qui a connu ces dernières années une transformation sans précédent. Étant donné que la traduction est tributaire des changements évoqués, au cours de deux premières décennies du XXIe siècle, on assiste 
à une redéfinition des pratiques de la traduction théâtrale et, ce qui s'ensuit, à une révision du rôle du traducteur de théâtre et de son activité. De nos jours, les méthodes de traduction des spectacles en direct, comme le surtitrage, bousculent la perception traditionnelle de la traduction et mettent en question les notions-clés qui se trouvent au coeur de la réflexion traductologique dès sa naissance, telles que le traducteur, le destinataire ou même le texte. Le présent article décrit les enjeux du surtitrage à la lumière des théories modernes de la de traduction théâtrale. En nous basant sur les ouvrages théoriques choisis, les voix des chercheurs et des professionnels du théâtre, nous nous pencherons d'abord sur le problème du statut et des rôles du traducteur théâtral, pour passer, dans la suite de l'article, à la spécificité $\mathrm{du}$ texte et de son contexte de production et de réception et les paradoxes qui en découlent.

\section{LE SURTITRAGE - MÉTHODE DE TRADUCTION EN PLEIN ESSOR}

De nos jours, de plus en plus souvent le transfert linguistique au théâtre prend la forme de traduction des spectacles en direct et en synchrone avec le jeu des comédiens. Ainsi, les méthodes comme l'interprétation simultanée (à l'aide des casques distribués aux spectateurs) ou le surtitrage, permettent au public étranger d'assister au spectacle simultanément avec celui qui connaît la langue du plateau.

Quant au surtitrage (à ne pas confondre avec le sous-titrage des émissions filmiques), cette méthode de traduction en direct des pièces de théâtre, des opéras et d'autres types de spectacles, consiste à projeter, généralement au-dessus de la scène, des fragments de la traduction qui sont nécessaires à une bonne compréhension de la pièce jouée. Les premiers surtitres ont été lancés à la Canadian Opera Company de Toronto pour «Elektra » de Richard Strauss, le 21 janvier 1983, par Gunta Dreifelds, Lotfi Mansouri et John Leberg. Les surtitres étaient alors affichés au moyen d'un projecteur à diapositives. Au cours de trente dernières années, cette méthode de traduction a été développée grâce aux technologies informatiques pour mieux répondre aux besoins des metteurs en scène et du public. Par conséquent, aujourd'hui, comme le notent les auteurs du « Guide du sur-titrage au théâtre » (Bataillon, Muhleisen \& Diez, 2016), le surtitrage devient une nécessité pour tous ceux qui souhaitent présenter leurs spectacles au public étranger :

Lorsqu'une institution, théâtre ou festival, prend la décision d'inviter un metteur en scène et une compagnie non francophone, elle n'envisage plus aujourd'hui de présenter leur spectacle sans avoir recours à un sur-titrage - que d'ailleurs le public espère, au double sens de souhaite et attend. Le sur-titrage n'apporte pas l'assurance d'une bonne fréquentation, mais son absence la compromettrait. Il fait désormais partie de l'économie des tournées internationales (Bataillon, Muhleisen \& Diez, 2016, p. 17). 


\section{SURTITRAGE FACE AUX THÉORIES MODERNES DE LA TRADUCTION THÉÂTRALE}

Une des plus grandes théoriciennes du théâtre, Anne Ubersfeld, dans son ouvrage majeur intitulé «Lire le théâtre I» (1978) invoque le caractère paradoxal d'art théâtral :

Le théâtre est un art paradoxal. On peut aller plus loin et y voir l'art même du paradoxe, à la fois production littéraire et représentation concrète ; à la fois éternel (indéfiniment reproductible et renouvelable) et instantané (jamais reproductible comme identique à soi) [...]. Paradoxe : art du raffinement textuel, de la plus haute et de la plus complexe poésie, d'Eschyle à Jean Genet en passant par Racine ou Hugo. Art de la pratique ou d'une pratique à gros traits, à grand signes, à redondances : il faut être vu, il faut être compris de tous. Ici encore l'écart se creuse entre le texte, qui peut être l'objet d'une lecture poétique infinie, et ce qui est de la représentation, immédiatement lisible (Ubersfeld, 1978, pp. 13-14).

Sans doute, ce caractère multidimensionnel, même insaisissable de l'art théâtral reste l'une des causes principales de la marginalisation de la traduction théâtrale au sein des sciences de traduction. Comme le fait remarquer Fabio Regattin : " beaucoup d'encre a été versé sur la traduction littéraire ou poétique, alors que la traduction théâtrale est restée dans l'ombre [...] La plupart des chercheurs persistent à déplorer une absence d'intérêt pour la matière » (2004, p. 156). Anne-Carole Upton et Terry Hale expliquent les raisons du faible intérêt que la traduction théâtrale suscite parmi les traductologues d'une manière suivante : " the complexity and inconstancy of the performance text clearly pose an enormous (and, so far, insuperable) challenge to translation theorists $\gg$ (Upton \& Hale, 2000, p. 9). À titre d'exemple, en France la première théorisation de la traduction théâtrale n'est parue qu'en mars 1982 dans la revue "Théâtre/Public » qui y a consacré son numéro spécial (44) réalisé sous la direction de Georges Banu et intitulé « Traduction ».

« La première contradiction dialectique de l'art théâtral, qui n'est pas sans incidence sur la traduction, est l'opposition entre le texte et la représentation » (Czubińska, 2016, p. 97). Elle a été expliquée par le sémioticien du théâtre, le chercheur anglais Keir Elam d'une manière suivante :

Le dramatic text (par la suite, texte dramatique ${ }^{1}$ ) est le texte écrit. Il n'existe qu'en point de départ au performance text (par la suite, texte spectaculaire), réalisation sur scène du texte dramatique. Le texte théâtral est la somme du texte dramatique et du texte spectaculaire (Elam, 1980, as cited in Regattin, 2004, p. 157).

Une telle vision duelle du texte au sein du spectacle, a permis au chercheur italien Fabio Regattin (2004) de catégoriser les théories modernes de la traduction

\footnotetext{
${ }^{1}$ Nous soulignons.
} 
théâtrale en quatre catégories, selon la place qui y est accordée au texte et à la représentation. Ainsi, il est possible de distinguer quatre catégories des théories de la traduction théâtrale modernes, à savoir :

a) les théories littéraires,

b) les théories basées sur le texte dramatique,

c) les théories basées sur le texte spectaculaire,

d) les théories dites néolittéraires.

«La première catégorie regroupe les études qui assimilent le texte de théâtre à un texte littéraire » (Regattin, 2004, p. 158). Ses nombreux partisans affirment que l'oralité, le rythme, la vocalité, la corporalité ou les idiolectes des personnages ne sont pas spécifiques au texte dramatique car on peut retrouver les mêmes traits dans la prose, notamment dans les dialogues de roman. "Dans cette perspective, la distinction entre traduction littéraire et traduction dramatique perd sa signification » (Regattin, 2004, p. 159) et la tâche du traducteur reste purement littéraire.

La deuxième catégorie, celle des théories basées sur le texte dramatique, comprend les théories dont les auteurs affirment que le texte de théâtre possède certaines caractéristiques qui lui sont propres, et, par contrecoup, exige une approche traductive adaptée. On pourrait citer non seulement les didascalies qui constituent le trait distinctif de l'écriture dramatique, mais aussi l'immédiateté de la réception du dialogue théâtral (il devient impossible au traducteur d'expliquer p. ex. les éléments culturels). Qui de plus est, le texte dramatique doit avoir l'influence immédiate sur le spectateur, susciter ses émotions et l'inciter à la réflexion. Enfin, le traducteur doit veiller sur la «jouabilité » de la pièce traduite, garder sa dimension orale et musicale. Tous ces arguments permettent aux partisans de cette approche d'envisager la traduction théâtrale comme une activité à part qui demande des compétences spécifiques et une approche distincte.

La troisième catégorie regroupe les théoriciens de la sémiotique théâtrale l'approche ayant révolutionné le regard sur le spectacle et sa traduction. Les auteurs comme Patrice Pavis (1976), Anne Ubersfeld (1978), Susan Basnett (1985) ou Keir Elam (1980) considèrent le texte dramatique du point de vue de son intégration dans la performance et son réseau sémiotique complexe :

A theatre text exists in a dialectal relationship with the performance of that text. The two texts - written and performed - are coexistant and inseparable, and it is in this relationship that the paradox for the translator lies (Basnett, 1985, p. 87).

Puisque la traduction y est considérée dans les catégories d'une réécriture dramaturgique, le traducteur devrait travailler en étroite collaboration avec le metteur en scène et avec les comédiens, participer aux répétitions du spectacle et être prêt à changer son texte selon les exigences de la mise en scène concrète. Jacques Lasalle va encore plus loin et présente la démarche que nous pouvons nommer: « une 
traduction égale une représentation" (Lasalle, 1982, as cited in Regattin, 2004). Toutes les théories basées sur la sémiotique « valorisent d'une façon considérable la traduction comme activité et aussi le rôle du traducteur qui cesse d'être une vitre transparente et devient l'un des auteurs de la mise en scène et du succès de la pièce » (Czubińska, 2016, p. 117).

La représentante de la dernière catégorie (les théories néolittéraires) - Susan Bassnett qui a complètement changé son opinion sur la traduction théâtrale au cours de ses recherches, conclut que le traducteur de théâtre devrait adopter en face d'un texte dramatique, les mêmes démarches que pour la traduction littéraire (1998, p. 105). En ce sens, son article « Still Trapped in the Labyrinth» (1998) s'oppose radicalement non seulement à la conception de Lassalle (une mise en scène égale une seule traduction), mais aussi à sa propre opinion d'il y a 13 ans. Selon Basnett : « une traduction qui respecte les difficultés et les ambiguïtés du texte original permet ainsi un nombre virtuellement indéfini des mises en scène. Ce n'est plus au traducteur de tenir compte des mises en scène potentielles, mais aux professionnels de la scène » (Regattin, 2004, p. 164).

$\mathrm{Vu}$ ce qui précède, il conviendrait de se poser la question : qu'en est-il du surtitrage ? Il est évident que les approches qui sont nées dans la plupart de cas, dans les années 80. et 90. du XXe siècle, n'ont pas prévu l'apparition des méthodes de traduction des spectacles en direct. D'où la nécessité de dégager les caractéristiques des nouveaux types de traduction afin de trouver un nouveau cadre théorique. Néanmoins, les théories de la traduction théâtrale traditionnelles, non seulement peuvent devenir une riche source d'inspiration, mais elles constituent un bon point de départ pour une discussion sur le statut des pratiques du surtitrage, la place qui y occupe le traducteur et le texte dramatique.

\section{TRADUCTEUR/SURTITREUR/OPÉRATEUR...}

Autant il est possible de citer de nombreuses définitions de la traduction, envisageant cette opération dans les catégories du transfert purement linguistique, culturel ou social, autant la définition du traducteur ne semble pas susciter tant de controverses. Le dictionnaire Trésor de la Langue Française nous précise que le traducteur est « une personne effectuant des traductions, transposant des textes d'une langue dans une autre », tandis que l'interprète : « une personne qui traduit les paroles d'un orateur, ou le dialogue de deux ou plusieurs personnes ne parlant pas la même langue et qui leur sert ainsi d'intermédiaire $»^{2}$. Toutefois, comme nous allons

\footnotetext{
${ }^{2}$ Les deux définitions viennent du dictionnaire Trésor de la Langue Française informatisé. Leurs adresses respective : http://atilf.atilf.fr/dendien/scripts/tlfiv5/advanced.exe?8;s=2724053310; http://atilf.atilf. fr/den dien/scripts/tlfiv5/search.exe?38;s=2724053310; cat=0;m=interpr\%8Ate.
} 
le démontrer dans ce chapitre, dans le contexte théâtral ni le statut, ni le nom, ni le rôle, ni les compétences du « traducteur » ne répondent aux catégories traditionnellement définies.

Tout d'abord, le travail sur la version surtitrée du spectacle se déploie en plusieurs étapes. Bruno Péran et Agnès Surbezy dans leur article intitulé: « Surtitrage et Langue des Signes : l'expérience d'une complicité ? » (2010) en distinguent deux : le volet littéraire et le volet technique, tandis que les auteurs du «Guide du surtitrage au théâtre " (Bataillon, Muhleisen \& Diez, 2016) proposent un modèle de travail qui comprend trois étapes, celle de la traduction du texte dramatique, celle de découpage et celle de "topage». Malgré ces nettes différences, les chercheurs et praticiens de cette méthode de traduction se mettent tous d'accord sur le caractère interdisciplinaire du processus d'élaboration des surtitres.

Bien que la première phase du travail, qui consiste en traduction du scénario de la pièce, ressemble fortement à la traduction littéraire, il n'en reste pas moins que le texte issu de cette étape initiale soit ultérieurement modifié pour répondre aux besoins de la représentation. Très souvent, ce sont les traducteurs littéraires professionnels auxquels les metteurs en scène confient la tâche de traduction du scénario de la pièce. Tel est le cas du metteur en scène polonais, Krzysztof Warlikowski, qui fait traduire ses spectacles en français par Margot Carlier, la traductrice connue de la littérature polonaise.

On pourrait oser dire que cette étape préliminaire de travail est la seule qui présente des traits qui relèvent du métier du traducteur, car la phase suivante - celle de découpage ou de séquençage du texte demande beaucoup plus de compétences informatiques que traductives. Ainsi, la traduction qui accompagnera le spectacle sous forme des surtitres doit être découpée en plusieurs séquences et ensuite saisie par un logiciel informatique. Le traducteur/surtitreur, au cours de son travail, doit prendre en considération plusieurs contraintes, parmi lesquelles on peut citer celle de temps d'affichage d'un surtitre (3-4 secondes) et celle d'espace ( 2 lignes, dont chacune de 45 caractères au maximum) ce qui entraîne la sélection et la condensation $\mathrm{du}$ contenu. Afin d'adapter la première version des surtitres au jeu des comédiens et au rythme du spectacle, le surtitreur est obligé de travailler avec un support visuel la captation vidéo du spectacle, et enfin, ce qui semble tout à fait naturel, assister aux répétitions. Selon Yvonne Griesel, cette nature hybride du travail du surtitreur efface les frontières entre la traduction et l'interprétariat car le surtitreur travaille en même temps avec le texte écrit (dramatic text) et le texte oral (performance text) (Griesel, 2009, p. 123).

Pendant cette étape, le surtitreur travaille aussi en collaboration avec le metteur en scène et avec l'équipe technique, non seulement pour adapter le texte du point de vue linguistique mais aussi technique. L'équipe de théâtre doit trouver la meilleure place pour l'affichage des surtitres (éléments de la scénographie, mur), le meilleur 
support (écrans led ou panneaux d'affichage) et le meilleur logiciel (PowerPoint ou les logiciels conçus pour ce type d'activité comme Maestro, Torticoli, Glypheo, Qstit, Spectitular). Nous voyons bien que toutes ces décisions dépassent le cadre des compétences traditionnel du traducteur.

Enfin, la dernière étape de travail laborieux sur les surtitres, que les praticiens appellent " le topage», est aussi celle qui risque de gâcher l'effet final car la moindre erreur de topage est immédiatement repérée par les spectateurs. Ce qui mérite d'être souligné, la régie des surtitres n'exige pas nécessairement de l'opérateur la maîtrise de la langue cible, car le «topeur » s'appuie sur la langue du spectacle. Vu qu'il dispose d'un scénario original accompagné des surtitres correspondants, c'est une maîtrise parfaite du spectacle et la rapidité de réaction et du sangfroid en cas d'oubli ou d'improvisation des comédiens qui deviennent nécessaires. Comme le note Laurence Van Goethem dans son article «L'art de surtitrer : voyages en langues étrangères »: "Les meilleurs surtitres sont les plus discrets, ceux qui coulent en douceur dans la scénographie et accrochent le moins possible l'œil du spectateur $\gg(2016)^{3}$.

Nous voyons donc à quel point les pratiques du surtitrage contribuent à la mise en question du statut du traducteur théâtral qui doit désormais assumer des rôles du traducteur littéraire/surtitreur/régisseur/topeur/opérateur selon l'étape de travail et la nomenclature. Anna Rędzioch-Korkuz dans son ouvrage intitulé « Opera surtitling as a Special Case of Audiovisual Tanslation. Towards a Semiotic and Translation Based Framework for Opera Surtitling » (2016) précise :

The process of surtitling may involve three persons in charge of the end-product, namely the translator, surtitler and operator. It may be done by one person who would carry out the whole process, wchich would match the essence of surtitling as a method of AVT. [...] Yet what it excludes is traditional understanding of the profession of translator as a person conducting transformations on two texts (2016, p. 118).

Si l'on admet qu'en raison de la spécificité de chaque étape de travail et des compétences requises, la version surtitrée du spectacle est élaborée par trois personnes différentes, laquelle portera alors le titre honorable de l'auteur des surtitres ? Quel nom devrait donc figurer sur les affiches du spectacle ? L'art de théâtre, qui est par définition collectif, ne permet pas de résoudre ce problème de manière claire et univoque. Sans doute, nous avons affaire à un nouveau métier qui demande des compétences qui relèvent de plusieurs domaines, celui de la traduction littéraire, en passant par la connaissance des arts scéniques jusqu'à l'informatique. Le nom conventionnel : " traducteur » ne convient pas à chaque étape de travail sur la version surtitrée d'un spectacle, car, comme nous l'avons démontré, l'opérateur ne doit

\footnotetext{
${ }^{3}$ La source de la citation : https://blog.alternativestheatrales.be/lart-de-surtitrer-voyages-en-lan gues-etrangeres/.
} 
même pas maitriser la langue cible du spectacle surtitré. Ainsi, le cas de cette nouvelle méthode de transfert linguistique prouve que les notions traditionnelles de traductologie nécessitent une révision.

\section{5. ...ET SON TEXTE}

Ayant analysé les ambiguïtés du travail du traducteur/ surtitreur, il est temps de se poser la question sur les caractéristiques du texte source et du texte cible. En revenant à la distinction de Keir Elam (1980) évoquée ci-dessus, il devient clair qu'en cas du surtitrage nous avons affaire au performance text (le texte spectaculaire) soit un texte multimodal, composé des signes de nature différente qui créent un réseau sémiotique :

La représentation est constituée par un ensemble de signes verbaux et non verbaux; le message verbal figure à l'intérieur du système de la représentation avec sa matière d'expression à lui, qui est acoustique (la voix). À quoi s'ajoutent tous les codes grâce auxquels peuvent être décodés les signes non verbaux, les codes visuels, musicaux, la proxémique, etc. (Ubersfeld, 1996, p. 23).

Nous pouvons en tirer la conclusion que « le texte traditionnel suppose une lecture selon l'ordre du temps tandis que la perception de la représentation est synchronique et suppose donc une organisation spatio-temporelle de signes multiples et simultanés » (Czubińska, 2016, p. 100). Toutes ces caractéristiques ont permis aux chercheurs comme Tomaszkiewicz (2006), Tryuk (2010), Dragnea (2010), Griesel $(2005,2009,2011)$ ou Rędzioch-Korkuz (2016), de considérer les surtitrage dans les catégories de la traduction audiovisuelle. Néanmoins, en raison d'un grand nombre de caractéristiques propres à la traduction des spectacles vivants, il faut considérer le surtitrage comme son type distinct.

Pour se rendre compte de la spécificité du transfert linguistique en cas du surtitrage, il faut souligner que, contrairement à la traduction théâtrale dite "conventionnelle », il ne s'agit pas de "traduire le texte destiné à la mise en scène, mais le texte issu de la mise en scène [...]. Or, ce texte ne peut exister qu'a posteriori, à la suite du spectacle » (Péran, 2010, para. 5-7). Ainsi, « le texte source » ou « le texte de départ » englobe ici non seulement le texte du scénario de la pièce, mais aussi sa réalisation scénique. Même si la première étape du travail sur le surtitrage consiste, dans la plupart de cas, en traduction du scénario, une telle traduction «préliminaire » doit être ensuite confronté au spectacle vivant, au jeu scénique et à l'improvisation des comédiens. Il en résulte que : «le texte source pour le surtitrage est donc un texte écrit à plusieurs mains, joué à plusieurs bouches, mais c'est aussi, par définition, un texte qui n'a pas réellement de matérialité physique » (Péran, 2010, 
para. 7). Par conséquent, vu son ancrage dans la mise en scène concrète ${ }^{4}$, on y voit l'écho des théories basées sur le texte spectaculaire et sur la sémiotique.

C'est là où réside la différence majeure entre le surtitrage et son homologue cinématographique - le sous-titrage. Pendant que le traducteur des films travaille sur une version achevée et fixée de l'ouvrage, le surtitreur est confronté au texte dramatique ou plutôt un texte spectaculaire en évolution constante. On qualifie le théâtre d'art d'éphémère, et le surtitrage, comme méthode de traduction, reflète ce caractère dynamique, créatif et vivant de l'art théâtral. Péran précise à ce sujet : « Le surtitrage n'existe que le temps de la représentation, il est intimement lié à elle dans la mesure où il est élaboré en fonction d'une énonciation scénique précise. Par conséquent, il ne peut être réutilisé si la situation d'énonciation est différente » (Péran, 2010, para. 21).

Pour illustrer cette relation étroite entre la mise en scène et le surtitrage, nous nous servirons d'exemple du spectacle du metteur en scène Olivier Frljić intitulé « Turbofolk » qui a été présenté en Pologne dans le cadre du festival théâtral Malta Festival 2017. Vu l'annulation de la subvention accordée préalablement par le ministre de la culture polonais aux organisateurs du festival, le metteur en scène a refusé de venir à Poznań. Néanmoins, il a choisi de prendre la parole en ajoutant à son spectacle une scène-commentaire concernant cette situation délicate. Étant donné que les surtitres polonais et anglais pour la pièce ont été déjà élaborés, on a dû traduire au dernier moment la scène ajoutée au spectacle par le metteur en scène. Les cas pareils ne sont par rares, bien au contraire, ils font preuve de l'originalité de l'art théâtral. La situation évoquée montre aussi qu'afin de créer une version surtitrée de la pièce, la collaboration entre le metteur en scène, les comédiens et le traducteur/surtitreur devient nécessaire.

Il ne faut pas oublier que le contexte de réception de l'oeuvre théâtrale avec les surtitres définit aussi la forme du «texte cible». Linda Dewolf souligne que : «le surtitrage est un écrit éphémère, fait en principe pour être lu et non relu » (2003, para. 21). De ce fait, contrairement à la traduction théâtrale «conventionnelle », le surtitreur n'est pas censé rendre dans le texte cible les éléments qu'on appelle les virtualités théatrales ${ }^{5}$, ni l'oralité du texte source car les traits du texte dramatique, comme les didascalies, sont déjà concrétisés dans la représentation même. Ce type de transfert du code oral en code écrit (Tryuk, 2010) ou la traduction diagonale (Gottlieb, 1994) qui est propre à la traduction audiovisuelle, a de nombreuses conséquences.

\footnotetext{
${ }^{4}$ Pour en savoir plus sur l'inscription physique et symbolique des surtitres à la représentation, voir l'article de Bruno Péran intitulé « Le surtitrage et son con-texte source : vers une approche intégrative du surtitrage théâtral » (2016) disponible en ligne : http://revues.univ-tlse2.fr/lamaindethot/index.php?id=651.

${ }^{5}$ «Les virtualités théâtrales présentes dans le texte écrit ne s'épanouissent que dans le jeu de l'acteur. Il faut donc que ces virtualités soient inscrites dans le texte traduit pour que celui-ci donne naissance à du théâtre et non à du dialogue illustré » (Déprats, 1982, p. 47).
} 
Afin d'illustrer le phénomène en question, nous nous servirons d'exemple de la pièce «(A)pollonia » du metteur en scène polonais Krzysztof Warlikowski avec les surtitres français (2009).

\begin{tabular}{|c|c|c|}
\hline Scénario de la pièce originale & Transcription du jeu scénique & $\begin{array}{l}\text { Le surtitrage français } \\
\text { de la pièce }\end{array}$ \\
\hline $\begin{array}{c}\text { Apollo: Żegnaj, pałacu, } \\
\text { który tak ukochałem. } \\
\text { Żegnajcie. } \\
\text { Będzie mi was brakowało. } \\
\text { Będzie mi brakowało waszego } \\
\text { sposobu bycia, cywilizacji, } \\
\text { która mnie stworzyła, } \\
\text { Wariacji Goldbergowskich } \\
\text { granych przez Glenna Goulda, } \\
\text { wierszy Rimbaud... } \\
\text { Bardzo lubiłem wasze filmy, } \\
\text { zwłaszcza westerny. }\end{array}$ & $\begin{array}{c}\text { Apollo: Żegnaj, pałacu, który tak } \\
\text { ukochałem. } \\
\text { Żegnajcie. Będzie mi was } \\
\text { brakowało. } \\
\text { Będzie mi brakowało waszego } \\
\text { sposobu bycia, } \\
\text { waszej cywilizacji, } \\
\text { która mnie stworzyła, nie? } \\
\text { Będzie mi brakowało } \\
\text { Wariacji Goldbergowskich, } \\
\text { ale granych przez Glenna Goulda, } \\
\text { beedzie mi brakowało poezii } \\
\text { Rimbaud... Bẹdzie mi... czego mi } \\
\text { jeszcze będzie brakowało? Będzie } \\
\frac{\text { mi brakowało waszych filmów. }}{\text { No. Najbardziej to lubiłem }} \\
\text { westerny. }\end{array}$ & $\begin{array}{c}\text { Apollo : Adieu palais } \\
\text { que j'ai tant aimé ! // } \\
\text { Adieu. } \\
\text { Vous allez me manquer. // } \\
\text { Votre façon d'être // } \\
\text { votre civilisation } \\
\text { qui m'a crée // } \\
\text { Les Variations Goldberg } \\
\text { jouées par Glenn Gould // } \\
\text { et les poèmes de Rimbaud. } \\
\text { Tout ça va me manquer. // } \\
\text { J'aimais beaucoup vos films, } \\
\text { les westerns surtout //7. }\end{array}$ \\
\hline
\end{tabular}

Dans le monologue cité ci-dessus, le comédien (Adam Nawojczyk) qui joue le rôle du dieu Apollo se met à improviser, d'où le décalage du jeu scénique par rapport au scénario de la pièce. Dans les surtitres français, on observe l'élimination des traits d'oralité comme les répétitions ou les hésitations (éléments soulignés) qui résultent de l'improvisation du comédien et de son jeu scénique. Le comédien emploie en polonais le registre familier où les traits d'oralité sont particulièrement marqués. Quant aux surtitres français, le registre neutre employé par la traductrice Margot Carlier constitue une sorte de compromis. En effet, les surtitres doivent être lisibles et compréhensibles pour le public qui n'a que quelques secondes pour saisir leur sens et l'intégrer aux autres éléments du réseau sémiotique. C'est d'autant plus important que les éléments extralinguistiques du spectacle influencent aussi la réception du contenu linguistique des surtitres. Dans la scène analysée, le comédien

\footnotetext{
${ }^{6}$ Dans les extraits cités, les fragments soulignés présentent les traits de l'oralité et de l'improvisation qui marquent le décalage entre le texte joué sur la scène et le scénario. Les fragments marqués en gras mettent en évidence le décalage entre les surtitres français et le jeu scénique, notamment l'élimination des traits d'oralité et l'atténuation du registre. Les barres obliques dans la troisième colonne marquent le découpage du texte-cible en fonction des pauses dans le jeu scénique du comédien.

${ }^{7}$ Les barres obliques marques le découpage du texte en séquences qui forment les surtitres.
} 
se comporte d'une manière provocatrice - pendant son monologue, il se déshabille, pour enfin se mettre à nu. Grâce à l'emploi du registre standard dans les surtitres, l'élément linguistique n'absorbe pas l'attention des spectateurs et leur permet de se concentrer sur l'improvisation et les gestes du comédien. Ce court passage nous montre à quel point les surtitres se trouvent intégrés au réseau sémiotique du spectacle. Il est donc nécessaire, même impératif de les décrire en prenant en considération tous les aspects qui influencent la réception du spectacle dans son intégralité.

\section{TRADUCTION OU ORIGINAL?}

Aussi paradoxal que cela puisse paraître, l'art théâtral connaît des situations où le texte de la " traduction » (si l'on considère le surtitrage dans les catégories de traduction) (re)devient l'original. On y est confronté lorsque les pièces, le plus souvent celles qui font partie du canon mondial, dans leurs mises en scène en langues « régionales » sont accompagnées des surtitres en langue originale de l'œuvre :

La diversité des textes proposés au théâtre influence très certainement la manière de surtitrer. Le surtitrage dépend ainsi du degré de notoriété de la pièce. Les drames qui font partie du patrimoine littéraire mondial sont plus ou moins connus du public, ce dont doit tenir compte le traducteur. Ce qui peut poser problème, ce sont les drames présentés en traduction dans une langue étrangère qui sont retraduits vers la langue source (Griesel, 2011, p. 175).

Le meilleur exemple de ce cas particulier, ce sont les pièces de Shakespeare jouées dans les pays du monde entier avec les surtitres anglais. Un tel cas a eu lieu en 2012 en Pologne lorsqu'un des théâtres de Poznań - Teatr Polski, a décidé de jouer la pièce «Hamlet» avec les surtitrage en langue anglaise. La direction du théâtre a voulu attirer le public international qui résidait pendant ce temps-là dans la ville, à l'occasion du Championnat d'Europe de football 2012. Les réalisateurs ont décidé d'employer la version originale de la pièce Skakespirienne, découpée en surtitres d'une longueur moyenne entre 8 à 10 lignes, tandis que les comédiens ont joué la pièce dans sa version polonaise moderne - la traduction de Stanisław Barańczak datant de l'année 1990. Tout en voulant rester fidèles à la pièce canonique, les réalisateurs n'ont pas pris en considération la forme du texte original - sa littéralité, sa densité et le caractère archaïque de la langue. Par conséquent, le surtitrage s'est avéré inadapté aux besoins du public étranger qui n'a pas été composé uniquement des usages natifs de la langue de Shakespeare. Même si la traduction a tenu compte de la littéralité de l'œuvre originale, le texte des surtitres ne répondait pas au contexte de la réception immédiate et la lecture fragmentée pendant le spectacle. 


\section{CONCLUSION}

Dans le cas du théâtre, qui est un «art paradoxal», de nouvelles méthodes de traduction, notamment le surtitrage, d'un côté constituent un facteur important de son internationalisation, mais de l'autre côté, en raison de leur caractère interdisciplinaire, représentent un défi non seulement pour les praticiens mais aussi pour les chercheurs. Comme nous l'avons démontré, les caractéristiques du surtitrage dépassent largement le cadre conventionnel d'études traductologiques. Ainsi, le surtitrage devient une méthode de traduction qui non seulement s'inscrit dans le caractère multidimensionnel de l'art théâtral, mais aussi reflète ses paradoxes. De ce fait, au lieu de donner de simples réponses, on voit plutôt émerger de nouvelles questions. Comment donc appréhender et décrire le produit de cette méthode de transfert linguistique qui reste en mouvement constant, qui n'a pas de forme matérielle, qui ne possède pas un seul auteur mais constitue le résultat d'effort de nombreuses personnes au cours de plusieurs étapes de sa création? L'art du surtitrage prouve donc à quel point la traduction «devient un concept de plus en plus difficile à contenir dans les limites d'une simple définition » (Dragnea, 2010, p. 9).

À la lumière des théories de traduction présentées dans le présent article et face à l'essor des pratiques du surtitrage, il conviendrait de se poser aussi une autre question - celle concernant le futur de la traduction théâtrale. L'épanouissement des pratiques du surtitrage, peut-il mener à la disparition progressive de la traduction théâtrale traditionnelle? Sinon, quelle serait la relation qui s'établira entre les œuvres traduites d'une manière conventionnelle et les spectacles surtitrés ? Dans quelques années, seront-nous témoins de la disparition du métier du traducteur théâtral au profit d'un nouveau métier adapté aux exigences du public et des professionnels du théâtre?

Sans doute, la marginalisation de la traduction théâtrale en tant qu'objet de recherches ne facilite pas la tâche aux chercheurs. Pourtant le surtitrage, qui a été pendant des années « cantonné à une position périphérique, tant physiquement que symboliquement » (Péran, 2016, p. 1), semble aujourd'hui sortir de l'ombre pour devenir l'objet d'un nombre croissant d'études. Face à tous ces doutes, il conviendrait quand même d'admettre que grâce à l'apparition du surtitrage dans les années $80 \mathrm{du}$ $\mathrm{XXe}$ siècle, nous pouvons observer actuellement une des plus grandes révolutions dans le domaine de la traduction de théâtre. 


\section{RÉFÉRENCES BIBLIOGRAPHIQUES}

Bassnett, S. (1985). « Ways through the Labyrinth: Strategies and Methods for Translating Theatre Texts ». In T. Hermans (réd.), The Manipulation of Literature. Studies in Literary Translation (pp. 87-102). Londres/Sydney: Croom Helm.

Bassnett, S. (1998). « Still Trapped in the Labyrinth. Further Reflections on Translation and Theatre ». In S. Bassnett \& A. Lefevere (réd.), Constructing Cultures: Essays on Literary Translation (pp. 90-108). Clevedon : Multilingual Matters.

Bataillon, M., Muhleisen, L. \& Diez, Y.-P. (2016). « Guide du sur-titrage au théâtre. Maison Antoine Vitez ». Accessible sur le site https://www.maisonantoinevitez.com/telechargements/ guide-pdf.

Czubińska, M. (2016). La stylisation et ses enjeux dans la traduction du théâtre des minorités francophones canadiennes en polonais. Série : Dysertacje Wydziału Neofilologii UAM w Poznaniu; 22. Poznań : Wydział Neofilologii UAM w Poznaniu. Accessible sur le site https://repozytorium.amu.edu.pl/bitstream/10593/17538/1/Czubinska_9788394739812.pdf.

Déprats, J.-M. (1982). « Traduire Shakespeare pour le théâtre ». Théâtre/Public, 44, 45-48.

Dewolf, L. (2003). " La place du surtitrage comme mode de traduction et vecteur d'échange culturel pour les arts de la scène ». Recherches Théâtrales du Canada, vol. 24, no. 1, 92-108.

Dragnea, A. (2010). Surtitrage du dialogue dramatique dans la pratique théâtrale contemporaine. Iaşi : Editura Lumen.

Elam, K. (1980). The Semiotics of Theatre and Drama. London : Methuen.

Gottlieb, H. (1994). « Subtitling: Diagonal Translation ». Perspectives - Studies in Translatology, vol. 2, no. 1, 101-121.

Griesel, Y. (2005). « Surtitles and Translation. Towards an Integrative View of Theater Translation ». MuTra - Multidimensional Translation Conference Proceedings. Accessible sur le site http://www.euroconferences.info/proceedings/2005_Proceedings/2005_proceedings.html.

Griesel, Y. (2009). « Surtitling: Surtitles an other hybrid on a hybrid stage ». Trans, 13, 119-127. Accessible sur le site http://www.trans.uma.es/pdf/Trans_13/t13_119-127_YGriesel.pdf.

Griesel, Y. (2011). « Le surtitrage sur scène : un transfert linguistique hybride, pragmatique et artistique en même temps ». In A. Serban \& J.-M. Lavaur (réd.), Traduction et médias audiovisuels (pp. 171-182). Lille : Preses Universitaires du Septentrion.

Pavis, P. (1976). Problèmes de sémiologie théâtrale. Québec : Presses de l'Université du Québec.

Péran, B. (2010). "Éléments d'analyse de la stratégie de traduction mise en œuvre dans le surtitrage ». Traduire, 223, 66-77. Accessible sur le site http://traduire.revues.org/288.

Péran, B. (2016). "Le surtitrage et son con-texte source : vers une approche intégrative du surtitrage théâtral ». La main de Thôt, 4. Accessible sur le site http://revues.univ tlse2.fr/ lamaindethot/index.php?id=651.

Péran, B. \& Surbezy, A. (2010). « Surtitrage et Langue des Signes : l'expérience d'une complicité ? ». Traduire, 223, 78-88. Accessible sur le site http://traduire.revues.org/295.

Regattin, F. (2004). "Théâtre en traduction : un aperçu du débat théorique ». L'Annuaire théâtral : revue québécoise d'études théâtrales, 36, 156-171.

Rędzioch-Korkuz, A. (2016). Opera surtitling as a Special Case of Audiovisual Tanslation. Towards a Semiotic and Translation Based Framework for Opera Surtitling. Frankfurt am Main : Peter Lang Edition.

Surbezy, A. (2006). « Quand la traduction s'adapte à la scène : le surtitrage (Sangre lunar de J.S. Sinisterra et El grito de los espejos de M. Lobera) ». In S. Hibbs \& M. Martinez (réd.), Traduction, adaptation, réécriture dans le monde hispanique contemporain (pp. 170-178). Toulouse: Presses Universitaires du Mirail. 
Théâtre/Public (1982), no. 44, mars-avril. Traduire. Dossier réalisé sous la direction de Georges Banu.

Tomaszkiewicz, T. (2006). Przekład audiowizualny. Warszawa : Wydawnictwo Naukowe PWN.

Tryuk, M. (2010). « Co to jest thumaczenie audiowizualne? ». Przektadaniec, 20, 26-39.

Ubersfeld, A. (1978). Lire le théâtre. Paris : Éditions Sociales.

Ubersfeld, A. (1996). Lire le théâtre I. Paris : Belin.

Upton, C.-A. \& Hale, T. (2000). Moving Target: Theatre Translation and Cultural Relocation. Manchester/Northampton : St. Jerome.

Van Goethem, L. (2016). "L'art de surtitrer : voyages en langues étrangères ». Accessible sur le site https://blog.alternativestheatrales.be/lart-de-surtitrer-voyages-en-langues-etrangeres/. 